\title{
Effect of the potential well on vibrational scattering and the validity of SSH theory
}

\author{
Albert F. Wagner* and Vincent McKoy ${ }^{\dagger}$ \\ Arthur A. Noyes Laboratory of Chemical Physics, ${ }^{+}$California Institute of Technology, Pasadena, California 91109
}

(Received 27 January 1972)

\begin{abstract}
The vibrational de-excitation probability, $P_{10}$, is calculated quantum mechanically over a large energy range for models of three collision systems: $\mathrm{O}_{2}-\mathrm{O}_{2}, \mathrm{Cl}_{2}-\mathrm{Cl}_{2}$, and $\mathrm{Br}_{2}-\mathrm{Br}_{2}$. The vibrational de-excitation cross section, $\sigma_{10}$, is similarly calculated for the $\mathrm{Cl}_{2}-\mathrm{Cl}_{2}$ model. $P{ }_{10}$ and $\sigma_{10}$ are obtained for the Lennard-Jones intermolecular potential and three other "well-less" potentials designed to duplicate the scattering of the Lennard-Jones potential. The results emphasize the adiabatic nature of potentials with wells and indicate that the acceleration approximation for the effect of the well is not valid. The curves of $P_{10}$ and $\sigma_{10}$ as a function of initial translational energy are used to obtain exact collision numbers. These numbers are compared to the results of SSH theory. SSH theory is found to predict collision numbers with reasonable accuracy except at low temperatures. SSH theory is also not suitable for analyzing experimental collision numbers for the well depth potential parameter.
\end{abstract}

\section{INTRODUCTION}

Most collisions between neutral atoms and molecules are governed by attractive forces at large separations and repulsive forces at short separations. Hence the potential for the collision has an attractive well. For vibrational inelastic collisions of molecules with themselves or with atoms, the potential well is often assumed ${ }^{1}$ only to increase the relative translational energy of the collision by an amount equal to the well depth. This acceleration approximation is basic to SSH theory, ${ }^{1}$ which is widely used in analyzing the results of vibrational relaxation experiments. There have been several studies ${ }^{1,2}$ of the accuracy of the acceleration approximation and of SSH theory, but they have employed approximate methods to obtain the necessary probabilities and cross sections. In this article we have analyzed through exact quantum mechanical calculations the effects of the Lennard-Jones well on models of three different inelastic vibrational collision systems: $\mathrm{O}_{2}-\mathrm{O}_{2}, \mathrm{Cl}_{2}-\mathrm{Cl}_{2}$, and $\mathrm{Br}_{2}-\mathrm{Br}_{2}$. We have also evaluated the accuracy of SSH theory both in predicting and analyzing experiments on these systems. Wilson ${ }^{3}$ has used exact quantum mechanical calculations to study the effects of the well on vibrational excitation. However, the collision systems he studied are quite unlike ours. Also, he did not evaluate the accuracy of SSH theory for his collision systems. This article is divided as follows. In Sec. I, we discuss our choice of collision systems and the method of modeling the collisions and in Sec. II, we describe the methods used to carry out the calculations. In Sec. III, we examine the probability of de-excitation by head-on collision, from the first excited to the ground vibrational state, $P_{10}$, as a function of initial translational energy, $E$. Calculations are done on all three systems for a Lennard-Jones intermolecular potential and for two other "well-less" potentials suggested by $\mathrm{SSH}$ theory. For the $\mathrm{Cl}_{2}-\mathrm{Cl}_{2}$ system, we examine the cross section for de-excitation, $\sigma_{10}$, as a function of $E$ for the same three potentials. In Sec. IV we examine for all three systems $P_{10}$ as a function of $E$ for the Lennard-Jones potential and for a modified "well-less" Lennard-Jones potential. This comparison best isolates the effects of the well. In Sec. V, we examine the accuracy of SSH theory for our three collision systems by comparing its predictions to those derived from the calculations in Sec. III. Section IV summarizes our results.

\section{SYSTEM MODELING}

The three collision systems $\mathrm{O}_{2}-\mathrm{O}_{2}, \mathrm{Cl}_{2}-\mathrm{Cl}_{2}$, and $\mathrm{Br}_{2}-\mathrm{Br}_{2}$ have increasing well depths in units of $\hbar \omega$, the vibrational gap of the diatom. Taken as a set, the three well depths cover the range of depths usually found in diatom-diatom or diatom-atom collisions. Collision systems with hard interactions, such as $\mathrm{H}_{2}-\mathrm{H}_{2}$, are not well represented by our set of systems. However, systems with hard interactions usually have small well depths, due to the tight binding of electrons about their molecular centers and their resulting low polarizability. We exclude hard collision systems from our study under the assumption that their well effects would be small. Wilson ${ }^{3}$ studied hard collision systems with large well depths. His study differs somewhat from ours and would not accurately model most collisions of simple molecules.

For the three systems of interest we wish to calculate the rotational averaged cross section for de-excitation from the first excited to the ground vibrational state, $\sigma_{10}$, as a function of $E$. The angular asymmetry of the actual intermolecular potential produces rotational as well as vibrational scattering. To obtain $\sigma_{10}$, we must sum over the properly weighted cross sections for each vibrational-rotational transition consistent with a $1 \rightarrow 0$ vibrational de-excitation. The spherically symmetric part of the intermolecular potential can produce only vibrational scattering. This potential is the result of 


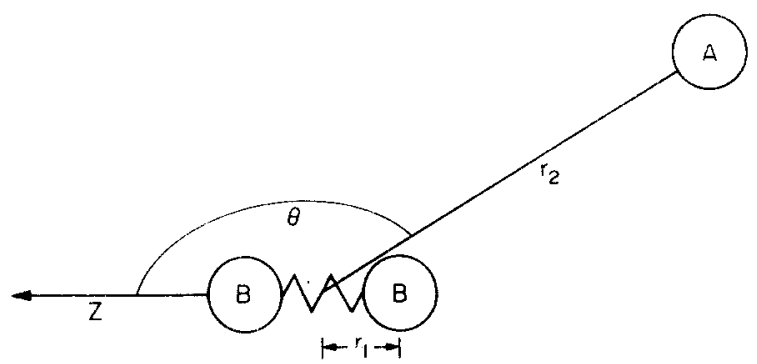

FIG. 1. Coordinate system.

rotationally averaging the full potential. It is frequently assumed that the vibrational cross sections obtained directly from the rotationally averaged potential accurately approximate the rotationally averaged vibrational-rotational cross sections obtained from the full potential. This has been shown ${ }^{4}$ to be true for low energy homonuclear diatom-atom collisions. We assume this is true here and use only spherically symmetric potentials. To further simplify our calculations, we will freeze one diatom's vibrational motion and approximate the other by a harmonic oscillator. The loss of half of the vibrational degrees of freedom and all of the anharmonicity of the actual collision system make our model quantitatively inaccurate. However, calculations ${ }^{5}$ on the $\mathrm{H}_{2}-\mathrm{H}_{2}$ collision system indicate that these last two approximations create no qualitative discrepancies for head-on collisions, where such discrepancies are most likely to occur. Also, most of our results will be based on the relative comparison of $P_{10}$ or $\sigma_{10}$ for different but related intermolecular potentials, so some of the quantitative inaccuracies must cancel out. Finally, since SSH theory is based on the same modeling described here, our calculations can then be used to test the accurcy of the theory.

Figure 1 shows our coordinate system. The initial direction of the frozen diatom, particle $A$, is the $z$ axis. The ball and spring picture for diatom $B-B$ actually represents the breathing sphere responsible for our spherically symmetric intermolecular potential, $r_{1}$ is the distance of one atom in B-B from the molecular center of mass (or the distance between the surface of the breathing sphere and its center). $\theta$ and $r_{2}$ describe the position of particle A relative to the center of mass of B-B. Since the potential is spherically symmetric,

TABLE I. System parameters. ${ }^{\mathbf{a}}$

\begin{tabular}{lccl}
\hline \multicolumn{1}{c}{ Parameter } & $\mathrm{O}_{2}-\mathrm{O}_{2}$ & $\mathrm{Cl}_{2}-\mathrm{Cl}_{2}$ & $\mathrm{Br}_{2}-\mathrm{Br}_{2}$ \\
\hline$\epsilon$ & 0.0508 & 0.441 & 1.106 \\
$\sigma$ & 131.7 & 141.7 & 168.1 \\
Unit of energy: $\hbar \omega\left({ }^{\circ} \mathrm{K}\right)$ & 2230 & 810 & 470 \\
Unit of length: & & & \\
$\quad \frac{1}{2}\left(\hbar \omega / k_{e}\right)^{1 / 2}(\AA)$ & 0.02606 & 0.02909 & 0.02538
\end{tabular}

a K. F. Herzfeld and T. A. Litovitz, Absorption and Dispersion of Uttrasonic Waves (Academic, New York, 1959), p. 321. there is no out-of-plane scattering and consequently no need for an azimuthal angle to describe the position of A. Since $A$ is a frozen $B-B$, its mass, $m A$, is twice $m_{B}$, the mass of $B$. In reduced units, ${ }^{6}$ the Hamiltonian $\mathfrak{F}$ for the $l$ th partial wave is

$$
\begin{aligned}
\mathfrak{H}=-(2 M)^{-1}\left(\partial^{2} / \partial r_{2}{ }^{2}\right)+ & {\left[l(l+1) / 2 M r_{2}{ }^{2}\right] } \\
- & -\frac{1}{2}\left(\partial^{2} / \partial y^{2}\right)+\frac{1}{2} y^{2}+V\left(r_{2}-r_{1}\right),
\end{aligned}
$$

where $M=m \mathrm{~A} /\left(m_{\mathrm{A}}+2 m_{\mathrm{B}}\right)=0.5$. The units of energy and length are $\hbar \omega$ and one-half the classical ground state vibrational amplitude. The diatom's displacement from equilibrium is $y$, while $V\left(\boldsymbol{r}_{2}-\boldsymbol{r}_{1}\right)$ is the intermolecular potential. We select the Lennard-Jones intermolecular potential for $V\left(r_{2}-r_{1}\right)$,

$$
V\left(r_{2}-r_{1}\right)=4 \epsilon\left\{\left[\sigma /\left(r_{2}-r_{1}\right)\right]^{12}-\left[\sigma /\left(r_{2}-r_{1}\right)\right]^{6}\right\} .
$$

This potential is qualitatively correct, is most frequently used by experimentalists, and is the potential assumed by SSH theory. In Table I we list $\epsilon, \sigma$, and the

TABLE II. The number of channels in the expansion set as a function of the number of open channels.

\begin{tabular}{cccc}
\hline \hline Open channels & $\mathrm{O}_{2}-\mathrm{O}_{2}$ & $\mathrm{Cl}_{2}-\mathrm{Cl}_{2}$ & $\mathrm{Br}_{2}-\mathrm{Br}_{2}$ \\
\hline 2 & 3 & 3 & 5 \\
3 & 4 & 4 & 5 \\
4 & 4 & 4 & 5 \\
5 & 4 & 4 & 5 \\
7 & 5 & 5 & 6 \\
9 & 5 & 6 & 6 \\
12 & 6 & 6 & 7 \\
14 & 8 & 7 & 7 \\
\hline \hline
\end{tabular}

reduced units of energy and length for our systems. The values are those of Herzfeld et al. ${ }^{1}$ These parameters are typical of nonhydrogenic collision systems.

The potential well should influence head-on collisions more strongly than glancing ones. Consequently, the effect of the well should be more pronounced in $P_{10}$ than in $\sigma_{10}$. For this reason and for reasons of economy, we calculated $P_{10}$ as a function of $E$ for all three systems, while calculating $\sigma_{10}$ as a function of $E$ for only the $\mathrm{Cl}_{2}-$ $\mathrm{Cl}_{2}$ system. The range of $E$ for all calculations was from 0 to 12 reduced units ( $E$ will always be given in reduced units).

\section{CALCULATIONAL METHODS}

We use the propagation method of Gordon ${ }^{7}$ to solve for ${ }^{l} \mathrm{~S}$, the $S$ matrix for the $l$ th partial wave. $P_{10}$ and $\sigma_{10}$ are related to ${ }^{l} \mathbf{S}$ by

$$
\begin{gathered}
P_{10}=\left|{ }^{0} S_{10}\right|^{2}, \\
\sigma_{10}=\left(\pi / K^{2}\right) \sum_{l}(2 l+1)\left|{ }^{l} S_{10}\right|^{2},
\end{gathered}
$$

where the wavenumber $K$ is $(2 M E)^{1 / 2}$. For our purposes, only the ${ }^{l} S_{10}$ element in ${ }^{\prime} S$ has to be accurate. 
In order to obtain an accurate ${ }^{l} S_{10}$ for our systems, considerably fewer than the total number of open channels need be included in the channel expansion at higher energies. This is due to the fact that at higher energies the excited motion described by the higher open channels is not actually executed by the system during de-excitation from the first excited to the ground state. In Table II, we compare the total number of open channels for all three collision systems to the number of channels which must be retained in the expansion to obtain $\left|{ }^{l} S_{10}\right|^{2}$ accurate to three or four digits in the third place. Since the calculation time increases as the cube of the number of channels in the expansion, a significant savings can be achieved by using less than the number of open channels at higher energies. It is generally true that calculations requiring only a part of the $S$ matrix need use only a channel set restricted about the scattering process of interest and that such

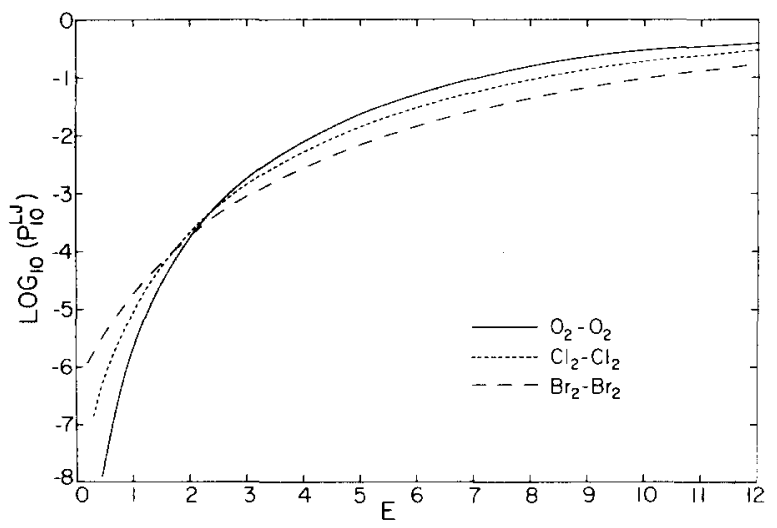

FIG. 2. $\log _{10}\left(P_{10} \mathrm{LJ}\right)$ vs $E$ for $\mathrm{O}_{2}-\mathrm{O}_{2}, \mathrm{Cl}_{2}-\mathrm{Cl}_{2}$, and $\mathrm{Br}_{2}-\mathrm{Br}_{2}$.

a channel set will often be smaller than the total number of open channels.

For the $\mathrm{Cl}_{2}-\mathrm{Cl}_{2}$ system, $\sigma_{10}$ must be calculated. At higher energies, $\sigma_{10}$ may require $\left|{ }^{l} S_{10}\right|^{2}$ for $l$ ranging from 0 to over 500 . However, since $\left|{ }^{l} S_{10}\right|^{2}$ is a smooth function of $l$, it can be readily interpolated. At each energy we calculate $\left|{ }^{l} S_{10}\right|^{2}$ for four to eight values of $l$ spanning the range of $l$ over which $\left|{ }^{l} S_{10}\right|^{2}$ is important. We concentrate the calculated values of $\left|{ }^{l} S_{10}\right|^{2}$ in regions where $\left|{ }^{l} S_{10}\right|^{2}$ is changing rapidly with l. These calculated elements are used to obtain the other values by interpolation. We actually interpolated $\log _{10}\left|{ }^{l} S_{10}\right|^{2}$ since this is a smoother function of $l$ than $\left|{ }^{i} S_{10}\right|^{2}$. The error in $\sigma_{10}$ due to interpolation with this coarse grid of calculated points is less than $5 \%$ except in the very low energy region, where the error could be as much as $10 \%$. Using an IBM $370 / 155$ computer and the procedures described in this section, we can calculate, for the $\mathrm{Cl}_{2}-\mathrm{Cl}_{2}$ system, $\sigma_{1}{ }^{0}$ from $E \approx 0$ to $E=12$ in roughly $10 \mathrm{~min}$ for one set of potential parameters. With procedures described elsewhere, ${ }^{4}$ elastic cross sections and elastic and inelastic differential

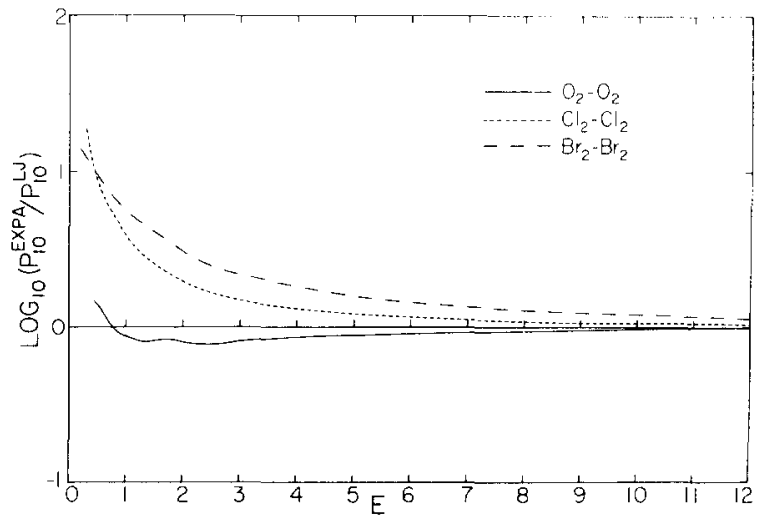

FIG. 3. $\log _{10}\left(P_{10}\right.$ EXPA $\left./ P_{10}{ }^{\mathrm{LJ}}\right)$ vs $E$ for $\mathrm{O}_{2}-\mathrm{O}_{2}, \mathrm{Cl}_{2}-\mathrm{Cl}_{2}$, and $\mathrm{Br}_{2}-\mathrm{Br}_{2}$

cross sections as a function of $E$ can also be obtained with a negligible increase in computer time. Approximate theories such as SSH theory are very useful, but they are no longer the only practical way to analyze or predict experimental results.

\section{LENNARD-JONES AND SSH INTER- MOLECULAR POTENTIALS}

All results obtained with the Lennard-Jones intermolecular potential are indicated by $\mathrm{LJ}$. Figure 2 shows $\log _{10}\left(P_{10}^{\mathrm{LJ}}\right)$ as a function of $E$ for all three systems. The three $P_{10}{ }^{\mathrm{LJ}}$ curves are featureless and very similar to each other. In general, $P_{10}{ }^{\mathrm{LJ}}$ is an oscillatory function of $E$. For our systems, the oscillations set in at $E>12$. For hydrogenic collisions, ${ }^{3,5}$ oscillations are evident by $E \approx 4$.

If the well serves only to accelerate the colliding species to an additional translational energy equal to its depth $\epsilon$, LJ should be replaceable by a potential whose value at large separations is $-\epsilon$. Such a potential boosts the incoming energy by $\epsilon$. The short range part of the potential should duplicate as much as possible the repulsive wall of $\mathrm{LJ}$. The replacement potential suggested by $\mathrm{SSH}$ theory has the form

$$
V\left(r_{2}-r_{1}\right)=H \exp \left[-\alpha\left(r_{2}-r_{1}\right)\right]-\epsilon .
$$

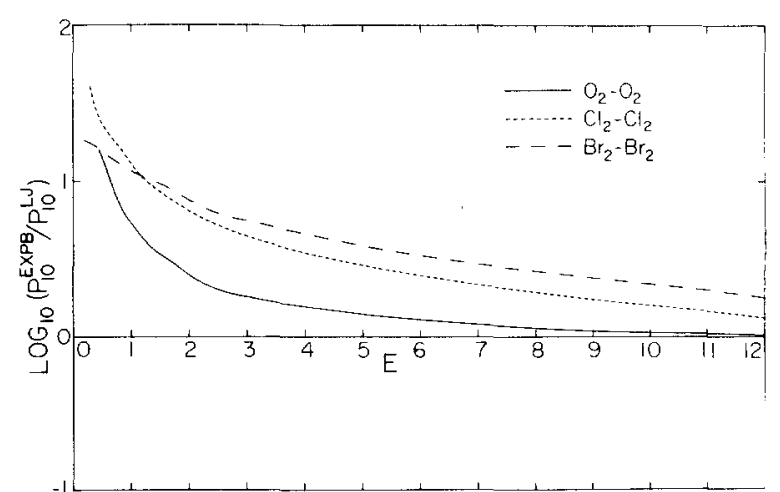

FIG. 4. $\log _{10}\left(P_{10} \mathrm{EXPB} / P_{10} \mathrm{LJ}\right)$ vs $E$ for $\mathrm{O}_{2}-\mathrm{O}_{2}, \mathrm{Cl}_{2}-\mathrm{Cl}_{2}$, and $\mathrm{Br}_{2}-\mathrm{Br}_{2}$. 


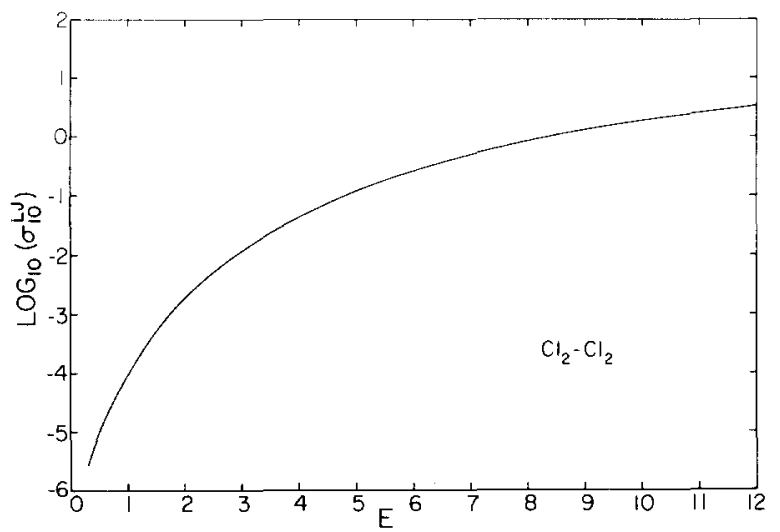

FIG. 5. $\log _{10}\left(\sigma_{10}{ }^{\mathrm{LJ}}\right)$ vs $E$ for $\mathrm{Cl}_{2}-\mathrm{Cl}_{2} \cdot \sigma_{10}{ }^{\mathrm{LJ}}$ is in square angstroms.

Here $H$ and $\alpha$ are functions of $E$, allowing a fit to LJ most appropriate for each value of $E$. There are two methods, $\mathrm{A}$ and $\mathrm{B}$, for determining $H$ and $\alpha$; we designate the two intermolecular potentials EXPA and EXPB and so label any results obtained with them.

In Figs. 3 and 4 , we plot $\log _{10}\left(P_{10}\right.$ EXPA $\left./ P_{10}^{\mathrm{LJ}}\right)$ and $\log _{10}\left(P_{10}{ }^{\mathrm{EXPB}} / P_{10}^{\mathrm{LJ}}\right)$, respectively, (for all three systems). At energies less than or comparable to $\epsilon, P_{10}$ EXPA differs from $P_{10}{ }^{\mathbf{L} J}$ by as much as an order of magnitude. At high energies $P_{10}{ }^{\text {EXPA }}$ is in close agreement with $P_{10}{ }^{\mathrm{LJ}}$. However, at these high energies the well has little influence on the colliding particles, because they are moving too fast to notice the well. Consequently, the possibility of the well being represented by a potential whose asymptotic value is $-\epsilon$ cannot be determined accurately at high energies. The good agreement of $P_{10}{ }^{\text {EXPA }}$ and $P_{10}{ }^{\mathrm{LJ}}$ in this region indicates only that the repulsive walls of the two potentials match closely. Similarly, the substantial disagreement of $P_{10}{ }^{E X P B}$ with $P_{10}{ }^{\mathrm{LJ}}$ even at high energies implies that EXPB fails to duplicate the repulsive wall of $\mathrm{LJ}$ for our systems. For this reason, no further calculations were done with EXPB.

In Fig. 5 we plot $\log _{10}\left(\sigma_{10} \mathrm{LJ}\right)$ as a function of $E$ for the $\mathrm{Cl}_{2}-\mathrm{Cl}_{2}$ system; $\sigma_{10}{ }^{\mathrm{LJ}}$ is in square angstroms. Over this

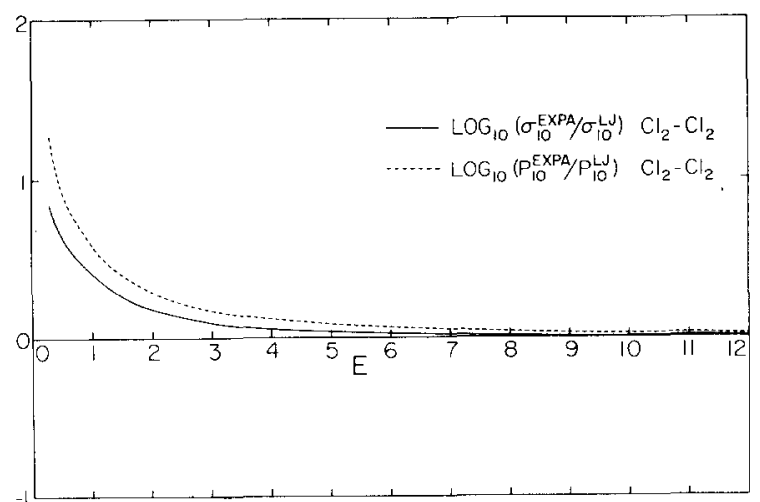

FIG. 6. $\log _{10}\left(\sigma_{10} \mathrm{EXPA} / \sigma_{10} \mathrm{LJ}\right)$ and $\log _{10}\left(P_{10} \mathrm{EXPA} / P_{10} \mathrm{LJ}\right)$ vs $E$ for $\mathrm{Cl}_{2}-\mathrm{Cl}_{2}$. energy range, $\sigma_{10}{ }^{\mathrm{LJ}}$ is very similar to $P_{10}^{\mathrm{LJ}}$ but roughly about an order of magnitude larger. In Fig. 6 we plot $\log _{10}\left(\sigma_{10}{ }^{\mathrm{EXPA}} / \sigma_{10}{ }^{\mathrm{LJ}}\right)$ for the $\mathrm{Cl}_{2}-\mathrm{Cl}_{2}$ system and replot $\log _{10}\left(P_{10}{ }^{\mathrm{EXPA}} / P_{10}{ }^{\mathrm{LJ}}\right)$ as a function of $E$. As expected, over the entire energy range $\sigma_{10}{ }^{\text {EXPA }}$ and $\sigma_{10}{ }^{\mathbf{L}}$ are in better agreement than $P_{10}{ }^{\mathrm{EXPA}}$ and $P_{10}{ }^{\mathrm{LJ}}$, because the well influences head-on collisions more strongly than glancing ones. Note that for $E$ greater than 2 or 3, the cross section for either LJ or EXPA is overestimated only by a factor of 3 or 4 by the relatively crude approximation

$$
\sigma_{10}=\pi r_{c}^{2} P_{10}
$$

where $r_{c}$ is the classical turning point. The physical interpretation of this approximation is that the hard sphere cross section, $\pi r_{c}^{2}$, is the cross section for all scattering processes while $P_{10}$ is the probability that a scattering process leads to de-excitation.

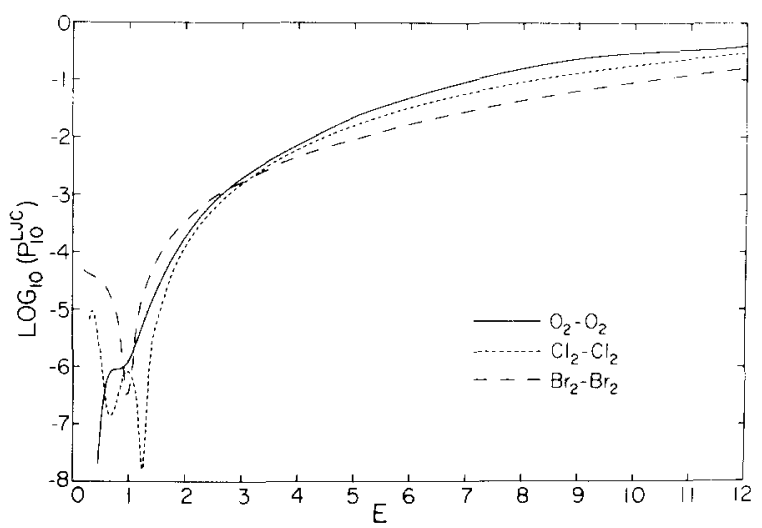

Fig. 7. $\log _{10}\left(P_{10} \mathrm{LJC}\right)$ vs $E$ for $\mathrm{O}_{2}-\mathrm{O}_{2}, \mathrm{Cl}_{2}-\mathrm{Cl}_{2}$, and $\mathrm{Br}_{2}-\mathrm{Br}_{2}$.

\section{THE "LENNARD-JONES CUTOFF" POTENTIAL}

Since EXPA approximates both the repulsive wall and the well of $\mathrm{LJ}$, the low energy disagreement of $P_{10}{ }^{\text {EXPA }}$ with $P_{10}{ }^{\mathrm{LJ}}$ may be due to both the limitations of fitting an exponentially repulsive potential to an inverse powers potential and the failure of representing the well entirely as an acceleration effect. The potential that is exactly $\mathrm{LJ}$ at small intermolecular separations and $-\epsilon$ at large separations would, in comparison with LJ, isolate the effects of only the well. Such a potential we call the Lennard-Jones cutoff potential (LJC), whose form is

$$
\begin{array}{rlrl}
V\left(r_{2}-r_{1}\right) & =4 \epsilon\left\{\left[\sigma /\left(r_{2}-r_{1}\right)\right]^{12}-\left[\sigma /\left(r_{2}-r_{1}\right)\right]^{6}\right\}, \\
& r_{2}-r_{1}<2^{1 / 6} \sigma \\
& =-\epsilon, & & r_{2}-r_{1} \geq 2^{1 / 6} \sigma,
\end{array}
$$

where the minimum of $\mathrm{LJ}$ occurs at $2^{1 / 6} \sigma$. In Fig. 7, we plot $\log _{10}\left(P_{10}^{\mathrm{LJC}}\right)$ as a function of $E$ for all three systems. The curves in Fig. 7 are quite unlike analogous curves for LJ, EXPA, and EXPB in that at low energies, 
$P_{10}^{\mathrm{LJC}}$ has structure for all three systems. Such structure could in general, come from two sources: The effects of several noninitial open or nearly open channels competing for the initial channel's amplitude or the interference effects of the wavefunction scattering off of several different parts of the potential. The first source may be considered responsible for subexcitation resonances and for the strong oscillations of all probability curves at high enough energy. The second source usually accounts for structure in only elastic scattering observables. However, Wilson ${ }^{3,8}$ has done vibrational scattering calculations with several potentials which have produced excitation probabilities with structure similar to ours over energy regions in which competitive effects seem unlikely. When we restrict the channel expansion of the wavefunction to only two channels at all energies and recalculate the curves shown in Fig. 7 , the structure at low energies is modified, but in no way removed. This seems to indicate that competitive effects are not as important as interference effects from several different regions of $L J C$. This is surprising because the potential with the most apparent structure is $\mathrm{LJ}$, yet it has no structure in its probability curves. An explanation for the structure in $P_{10}{ }^{\mathrm{LJC}}$ could be as follows. For all potentials, the region about the classical turning point is important in determining the scattering. For a monotonic repulsive potential, the tail of the potential would not usually be important in the scattering. We would suspect this to be true for EXPA. Suppose that for LJC the tail can effectively scatter. At low energies, tail scattering would dominate, while at high energies classical turning point scattering would dominate. In the intermediate region there would be interference. In $\mathrm{LJ}$, the effects of the tail of $\mathrm{LJC}$ are washed out by the climb out of the well the scattering wavefunction must undergo. The well makes $L J$ a long range, more adiabatic potential and allows the system to get used to and to get over the effects of the tail in LJC. To test this explanation, we can add on to LJC an exponential potential tail at $r_{2}-r_{1}=r_{0}$ where $\sigma \leq r_{0} \leq 2^{1 / 6} \sigma$. The value and slope of the exponential potential can be made to match LJC at $r_{0}$. If the

FIG. $8 . \log _{10}\left(P_{10} \mathrm{C}\right)$ vs $E$ for $\mathrm{O}_{2}-\mathrm{O}_{2}, \mathrm{Cl}_{2}-\mathrm{Cl}_{2}$, and $\mathrm{Br}_{2}-\mathrm{Br}_{2}$.

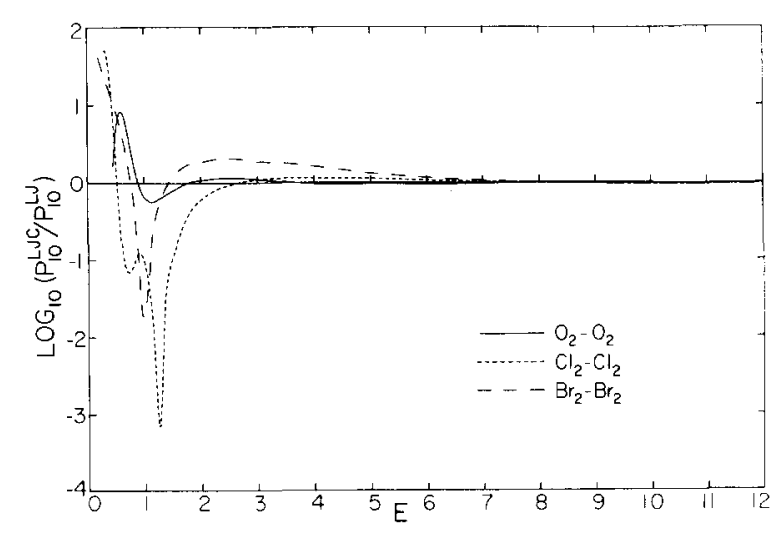

FIG. 9. $\log _{10}\left(P_{10} \mathrm{LJC} / P_{10} \mathrm{LJ}\right)$ vs $E$ for $\mathrm{O}_{2}-\mathrm{O}_{2}, \mathrm{Cl}_{2}-\mathrm{Cl}_{2}$, and $\mathrm{Br}_{2}-\mathrm{Br}_{2}$.

exponential tail is ineffective at scattering and if the explanation of the structure of $P_{10}{ }^{\mathrm{LJC}}$ is correct, the probabilities of this composite potential, $P_{10}{ }^{c}$, should be dominated by classical turning point scattering and show no structure. For the $\mathrm{O}_{2}-\mathrm{O}_{2}$ and $\mathrm{Cl}_{2}-\mathrm{Cl}_{2}$ systems, we set $r_{0}$ equal to $\sigma$; for the $\mathrm{Br}_{2}-\mathrm{Br}_{2}$ system, we set $r_{0}$ equal to the position where LJC has a value $-\epsilon / 2$. In Fig. 8 we plot $\log _{10}\left(P_{10}{ }^{C}\right)$ as a function of low energies for all three systems. Since there is no structure in $P_{10}{ }^{C}$, our explanation seems to be reasonable. In Fig. 9, we plot for all three systems $\log _{10}\left(P_{10}^{\mathrm{LJC}} / P_{10}^{\mathrm{LJ}}\right)$ as a function of $E$. The curves are dominated by the structure in $P_{10}{ }^{\mathrm{LJC}}$. However, outside this region of structure, the results in Fig. 9 indicate that increasing $E$ by the well depth overestimates the effect of the well. This conclusion and our explanation for the structure in $P_{10}$ LJC emphasize that potentials with wells have a longer range and therefore are more adiabatic than "well-less" potentials and any structure in the probabilities for "well-less" potentials is washed out.

\section{COMPARISONS WITH SSH THEORY}

SSH theory provides analytical expressions to determine the collision number $Z_{10}$, the average number of collisions a molecule undergoes before relaxing from the first excited to the ground state. If $\tau_{10}$ is the relaxation time and $\tau_{c}$ is the time between collisions, then

$$
Z_{10}=\tau_{10} / \tau_{c}
$$

If the system's relaxation is dominated by relaxation from the first to the ground state, then

$$
\tau_{10}=\left(k_{10}-k_{01}\right)^{-1}=k_{10}{ }^{-1}[1-\exp (-\hbar \omega / k T)]^{-1},
$$

where $k_{10}$ is the rate constant. If $k_{c}$ is the total rate at which scattering events take place per target molecule, then

$$
\tau_{c}^{-1}=k_{c} \text {. }
$$

For a one-dimensional system in translational equilib- 


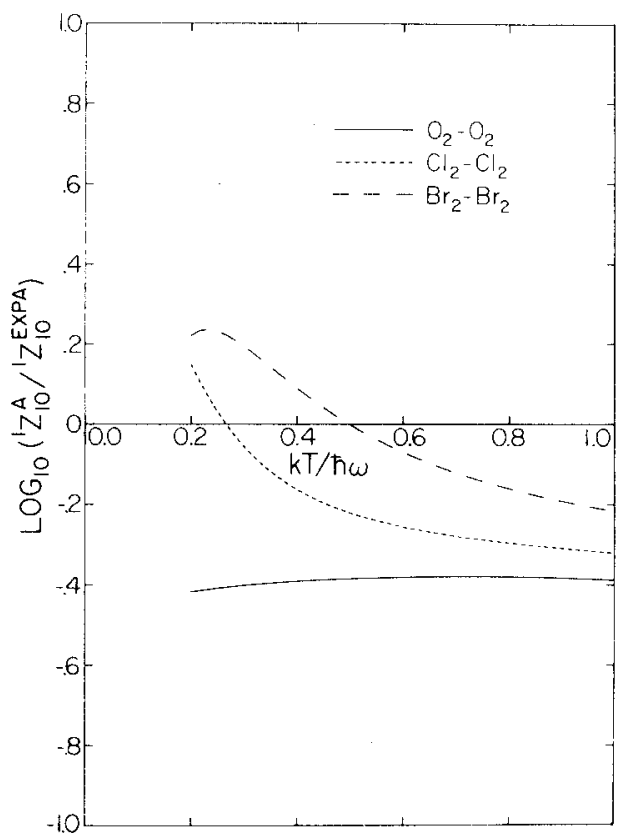

FIG. 10. $\log _{10}\left({ }^{1} Z_{10}{ }^{\mathrm{A}} /{ }^{1} Z_{10}{ }^{\mathrm{EXPA}}\right)$ vs $k T / \hbar \omega$ for $\mathrm{O}_{2}-\mathrm{O}_{2}, \mathrm{Cl}_{2}-\mathrm{Cl}_{2}$, and $\mathrm{Br}_{2}-\mathrm{Br}_{2}$.

rium, $k_{10}$ has the following form:

$$
k_{10}=\int_{0}^{\infty} P_{10}(E)\left(\frac{2}{M} E\right)^{1 / 2} d n(E) d E,
$$

where

$$
d n(E)=\rho(\pi k T)^{-1 / 2} E^{-1 / 2} \exp (-E / k T) .
$$

Here $\rho$ is the number of particles per unit length and

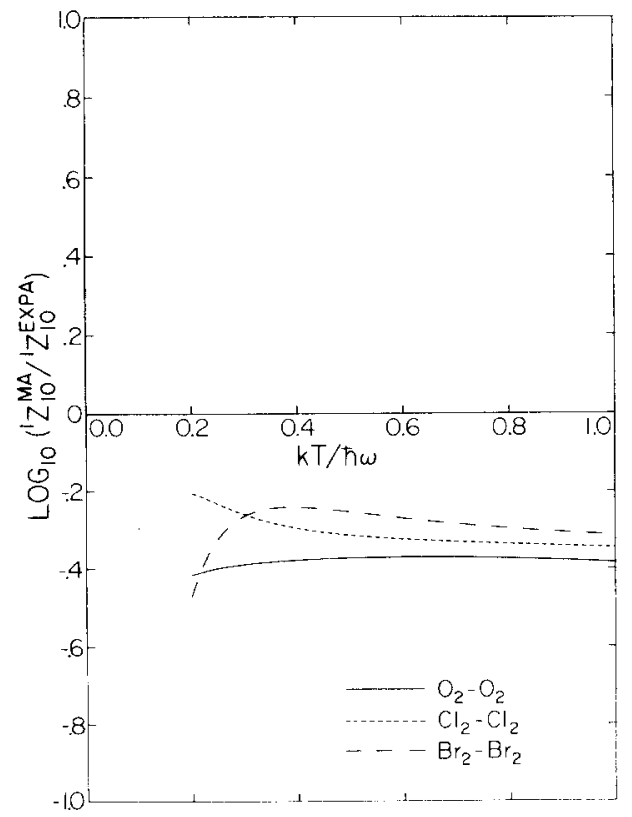

FIG. 11. $\log _{10}\left({ }^{1} Z_{10} \mathrm{MA} /{ }^{1} Z_{10}{ }^{\mathrm{EXPA}}\right)$ vs $k T / \hbar \omega$ for $\mathrm{O}_{2}-\mathrm{O}_{2}, \mathrm{Cl}_{2}-\mathrm{Cl}_{2}$, and $\mathrm{Br}_{2}-\mathrm{Br}_{2}$. $d n(E)$ is the Maxwell distribution of particles per unit length between energies $E$ and $E+d E$. For a onedimensional system, the probability that every encounter produces some scattering is 1 , for the encounters are all head on. Therefore,

$$
\begin{aligned}
k_{c} & =\int_{0}^{\infty}\left(\frac{2}{M} E\right)^{1 / 2} d n(E) d E \\
& =\rho(2 k T / \pi M)^{1 / 2} .
\end{aligned}
$$

Then the collision number for a one-dimensional system

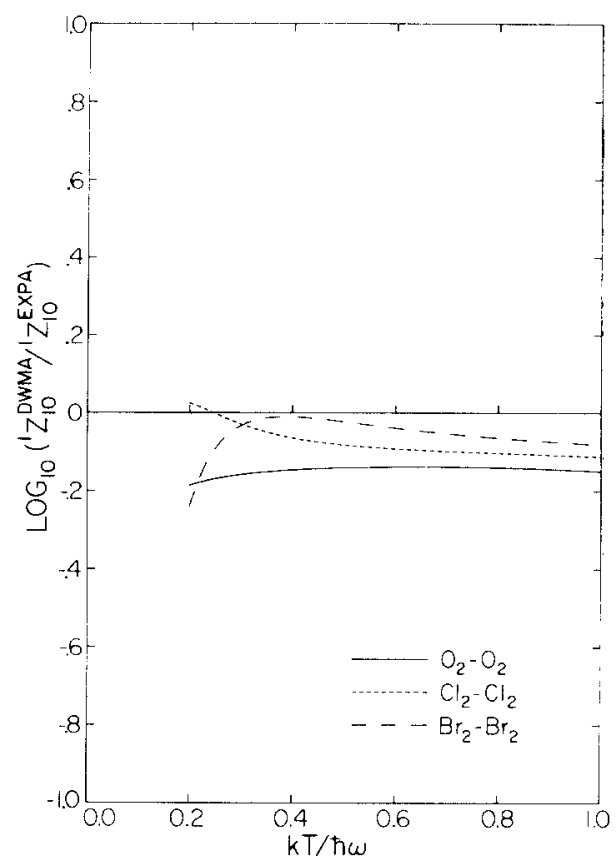

Fig. 12. $\log _{10}\left({ }^{1} Z_{10}\right.$ DWMA $\left./ 1 Z_{10}{ }^{\mathrm{EXPA}}\right)$ vs $k T / \hbar \omega$ for $\mathrm{O}_{2}-\mathrm{O}_{2}, \mathrm{Cl}_{2}-\mathrm{Cl}_{2}$, and $\mathrm{Br}_{2}-\mathrm{Br}_{2}$.

is

${ }^{1} Z_{10}=\left(1-e^{-\hbar \omega / k T}\right)^{-1}\left[(k T)^{-1} \int_{0}^{\infty} P_{10}(E) e^{-E / k T} d E\right]^{-1}$.

The superscript 1 on ${ }^{1} Z_{10}$ indicates a one-dimensional system. For a three-dimensional system in translational equilibrium, $k_{10}$ has the form,

$$
k_{10}=\int_{0}^{\infty} \sigma_{10}(E)\left(\frac{2}{M} E\right)^{1 / 2} d n(E) d E,
$$

where

$$
d n(E)=\rho 2 \pi(\pi k T)^{-3 / 2} E^{1 / 2} \exp (-E / k T) d E .
$$

Here $\rho$ is the number of particles per unit volume and $d n(E)$ is the Maxwell distribution of particles per unit volume between energies $E$ and $E+d E$. To determine $k_{e}$ we need an expression for the total cross section. 
Herzfeld $e t a l{ }^{1}$ implicitly write $k_{c}$ as

$$
\begin{aligned}
k_{c} & =\int_{0}^{\infty}(\text { total cross section })\left(\frac{2}{M} E\right)^{1 / 2} d n(E) d E \\
& \approx \sigma_{c} \int_{0}^{\infty}\left(\frac{2}{M} E\right)^{1 / 2} d n(E) d E \\
& =\sigma_{c} \rho(8 k T / \pi M)^{1 / 2} .
\end{aligned}
$$

Here $\sigma_{c}$ is an effective cross section which is temperature dependent. Since we will look at only ratios of collision numbers, the exact value of $\sigma_{c}$ is unimportant. The collision number for a three-dimensional system is

$Z_{10}=\left\{\left(1-e^{-\hbar \omega / k T}\right)^{-1} \sigma_{c}\left[\frac{1}{(k T)^{2}} \int_{0}^{\infty} \sigma_{10}(E) E e^{-E / k T} d E\right]\right\}^{-1}$.

SSH theory provides approximate formulas for the

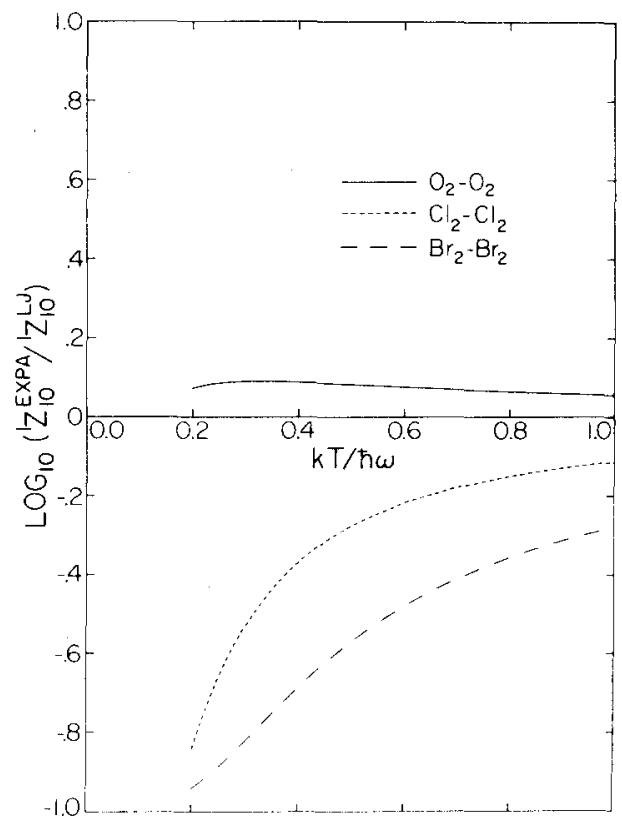

FIG. 13. $\log _{10}\left({ }^{1} Z_{10}{ }^{\mathrm{EXPA}} /{ }^{1} Z_{10}{ }^{\mathrm{LJ}}\right)$ vs $k T / \hbar \omega$ for $\mathrm{O}_{2}-\mathrm{O}_{2}, \mathrm{Cl}_{2}-\mathrm{Cl}_{2}$, and $\mathrm{Br}_{2}-\mathrm{Br}_{2}$.

bracketed terms in the Eqs. (13) and (16) for ${ }^{1} Z_{10}$ and $Z_{10}$. From the calculations presented in Sec. III, we can calculate these terms exactly. We first consider the onedimensional model of our three collision systems and then the three-dimensional model of the $\mathrm{Cl}_{2}-\mathrm{Cl}_{2}$ system.

The SSH formula for ${ }^{1} Z_{10}$ is based on the following three approximations used in evaluating:

$$
(k T)^{-1} \int_{0}^{\infty} P_{10}(E) e^{-E / k T} d E .
$$

First, LJ can be replaced by either EXPA or EXPB. We will discuss only the SSH formulas using EXPA. From the discussion of Sec. III, we would expect and

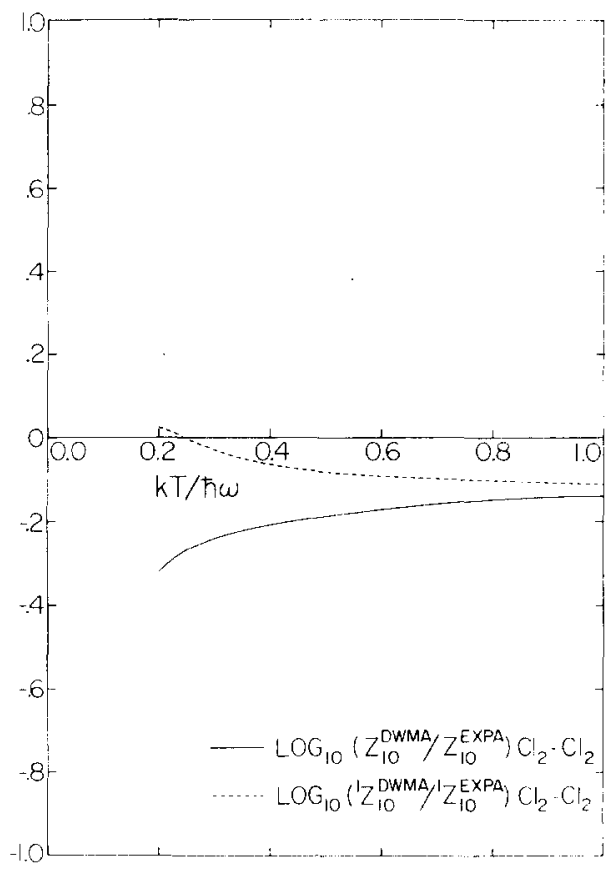

FIG. 14. $\log _{10}\left(Z_{10}\right.$ DWMA $/ Z_{10}$ EXPA $)$ and $\log _{10}\left({ }^{1} Z_{10}\right.$ DW MA $\left./{ }^{1} Z_{10}{ }^{\text {EXPA }}\right)$ vs $k T / \hbar \omega$ for $\mathrm{Cl}_{2}-\mathrm{Cl}_{2}$.

our calculations show that the SSH formulas using EXPB are not as accurate as those using EXPA. Second, $P_{10}$ can be replaced by the Jackson-Mott ${ }^{9}$ formula for $P_{10}{ }^{D W}$, the distorted wave probability for a head-on collision governed by an exponentially repulsive potential. Third, the integral of $P_{10}$ over the Maxwell distribution can be evaluated by a modified

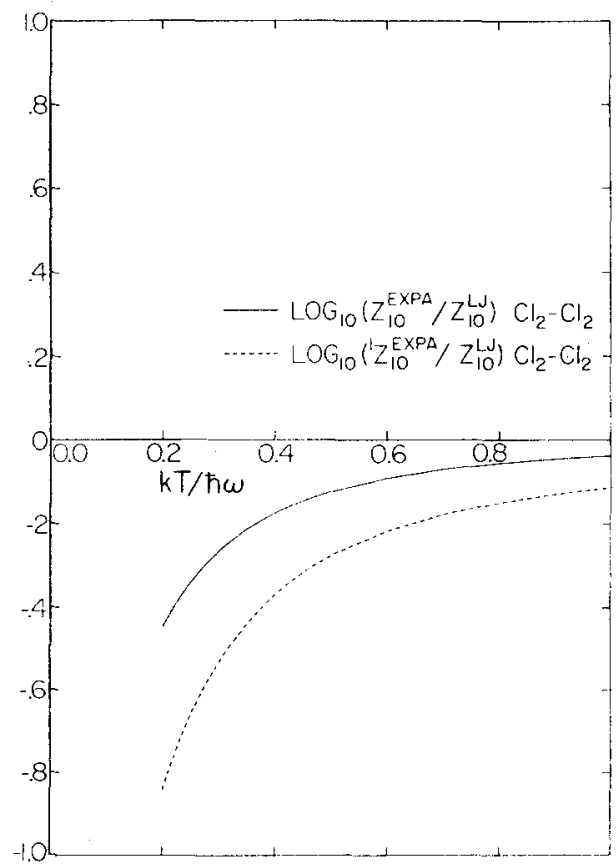

FIG. 15. $\log _{10}\left(Z_{10} \mathrm{EXPA} / Z_{10} \mathrm{~L} \mathrm{~J}\right)$ and $\log _{10}\left({ }^{1} Z_{10} \mathrm{EXPA} /{ }^{1} Z_{10}{ }^{\mathrm{L} J}\right)$ vs $k T / \hbar \omega$ for $\mathrm{Cl}_{2}-\mathrm{Cl}_{2}$. 


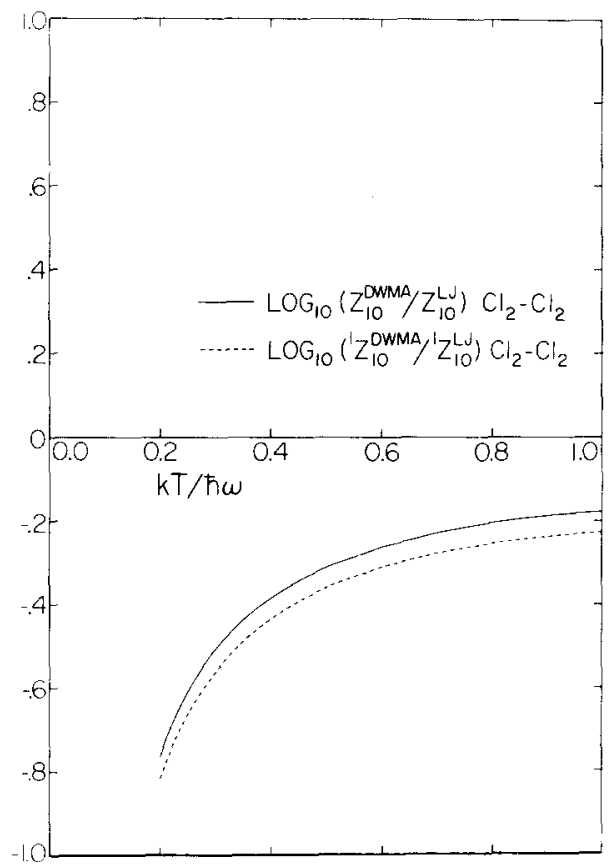

FIG. 16. $\log _{10}\left(Z_{10} \mathrm{DWMA} / Z_{10}^{\mathrm{LJ}}\right)$ and $\log _{10}\left({ }^{1} Z_{10} \mathrm{DWMA} /{ }^{1} Z_{10}^{\mathrm{LJ}}\right)$ vs $k T / \hbar \omega$ for $\mathrm{Cl}_{2}-\mathrm{Cl}_{2}$.

method of steepest descent. Let us designate ${ }^{1} Z_{10}{ }^{\mathrm{A}}$ as the SSH collision numbers for the EXPA fit to LJ. If the second and third approximations are exact, ${ }^{1} Z_{10}{ }^{A}$ should be identical to ${ }^{1} Z_{10}{ }^{\text {EXPA }}$. In Fig. 10 we plot for all three systems the $\log _{10}\left({ }^{1} Z_{10} \mathrm{~A} /{ }^{1} Z_{10}{ }^{\mathrm{EXPA}}\right)$ as a function of reduced temperature $k T / \hbar \omega$. One unit in reduced temperature is $2230^{\circ} \mathrm{K}$ for $\mathrm{O}_{2}-\mathrm{O}_{2}, 810^{\circ} \mathrm{K}$ for $\mathrm{Cl}_{2}-\mathrm{Cl}_{2}$, and $470^{\circ} \mathrm{K}$ for $\mathrm{Br}_{2}-\mathrm{Br}_{2}$. In Fig. $10,{ }^{1} Z_{10}{ }^{\mathrm{A}} /{ }^{1} Z_{10}{ }^{\mathrm{EXPA}}$ varies rapidly at low temperatures for two systems and at higher temperatures tends to a constant considerably less than 1 for all three systems. The second and third approximations are in error. Examining the third approximation first, we find that the evaluation of the integral in the expression (17) is obtained by expanding the integrand about its maximum value. In that expansion, the dependence of $\alpha$ on $E$ is ignored; $\alpha$ is one of the potential parameters in EXPA [see Eq. (5)]. Although $\alpha$ is a weak function of $E$, the Jackson-Mott formula for $P_{10} \mathrm{DW}$ is a very strong function of $\alpha$, especially at low energies. In the SSH formula $\alpha_{M}$ is used where $\alpha_{M}$ is the value of $\alpha$ at the energy, $E_{M}$, for which the integrand has a maximum. Let us use in the expansion of the integrand,

$$
\alpha=\alpha_{M}+\left(E-E_{M}\right) \alpha_{M}^{\prime},
$$

where $\alpha_{M}{ }^{\prime}=\partial \alpha / \partial E \mid E=E_{M}$. To first order in $\alpha_{M}{ }^{\prime}$, we get the modified collision number ${ }^{1} Z_{10}{ }^{\text {MA }}$,

${ }^{1} Z_{10}{ }^{M A}=\left(1-\frac{1}{3} \beta v_{M}\right) \exp \left\{[(1+2 \epsilon) / 2 k T] \beta v_{M}\right\} Z_{10}{ }^{A}$

where $\beta=\alpha_{M} / \alpha_{M} ; v_{M}=\left[(2 / M) E_{M}\right]^{1 / 2}$. $\beta$ can be determined from $\alpha_{M}$ and the formula relating $\alpha$ to $\epsilon$ and $\sigma$ for each value of $E$. In Fig. 11 we plot for all three systems $\log _{10}\left({ }^{1} Z_{10} \mathrm{MA} /{ }^{1} Z_{10} \mathrm{EXPA}\right)$ as a function of $k T / \hbar \omega .{ }^{1} Z_{10} \mathrm{MA} /$ ${ }^{1} Z_{10}{ }^{\text {EXPA }}$ does not vary rapidly with reduced temperature. The second approximation can also be improved because the Jackson-Mott formula for $P_{10} \mathrm{DW}$ is not as accurate as the exact formula for $P_{10}{ }^{\mathrm{DW}}$ evaluated by Mies. ${ }^{10}$ Secrest ${ }^{11}$ has shown that $P_{10}{ }^{D W}$ is quite close to $P_{10}{ }^{\text {EXPA }}$ for several systems. Let ${ }^{1} Z_{10}{ }^{\mathrm{DWMA}}$ designate the $\mathrm{SSH}$ collision number with both the second and third approximations improved upon. In Fig. 12 we plot for all three systems $\log _{10}\left({ }^{1} Z_{10} \mathrm{DWMA} /{ }^{1} Z_{10}{ }^{\mathrm{EXPA}}\right)$ as a function of $k T / \hbar \omega$. The ratio ${ }^{1} Z_{10}{ }^{\mathrm{DWMA}} /{ }^{1} Z_{10}{ }^{\mathrm{EXPA}}$ is approximately 1 over the whole temperature range. Therefore the second and third approximations suitably modified introduce little error into one-dimensional collision numbers. The effect of the first approximation is shown in Fig. 13 where $\log _{10}\left({ }^{1} Z_{10}{ }^{\mathrm{EXPA}} /{ }^{1} Z_{10}{ }^{\mathrm{LJ}}\right)$ for all three systems is plotted as a function of $k T / \hbar \omega$. The low energy disagreement of $P_{10}{ }^{\text {EXPA }}$ with $P_{10}{ }^{\mathrm{LJ}}$ discussed in Sec. III appears as a low temperature disagreement of ${ }^{1} Z_{10}{ }^{\text {EXPA }}$ with ${ }^{1} Z_{10}{ }^{\mathrm{LJ}}$. Figures $10-13$ show that, for a onedimensional model of our three collision systems, a modified form of SSH theory correctly predicts collision numbers except at temperatures low enough to make the effects of the well important.

The SSH formula for $Z_{10}$ is based on an approximate evaluation of

$$
\frac{1}{(k T)^{2}} \int_{0}^{\infty} \sigma_{10}(E) E e^{-E / k T} d E
$$

This requires a fourth approximation not used in

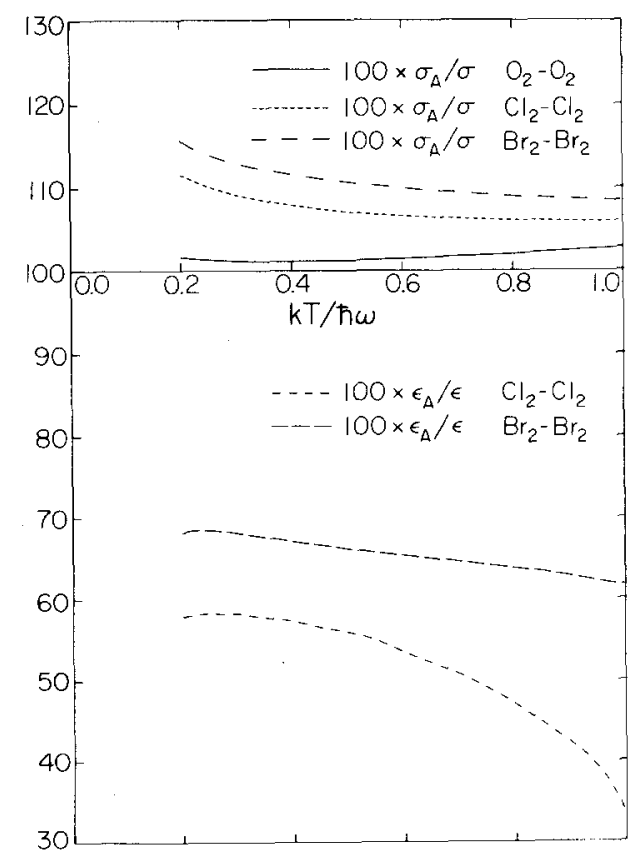

Fig. 17. $100 \times \sigma_{\Delta} / \sigma$ vs $k T / \hbar \omega$ for $\mathrm{O}_{2}-\mathrm{O}_{2}, \mathrm{Cl}_{2}-\mathrm{Cl}_{2}$, and $\mathrm{Br}_{2}-\mathrm{Br}_{2}$; $100 \times \epsilon_{A} / \epsilon$ vs $k T / \hbar \omega$ for $\mathrm{Cl}_{2}-\mathrm{Cl}_{2}$ and $\mathrm{Br}_{2}-\mathrm{Br}_{2}$. 
evaluating ${ }^{1} Z_{10}$, i.e.,

$$
\begin{aligned}
& {\left[\frac{1}{(k T)^{2}} \int_{0}^{\infty} \sigma_{10}(E) E e^{-E / k T} d E\right]} \\
& =\pi r_{M}^{2}\left[(k T)^{-1} \int_{0}^{\infty} P_{10}(E) e^{-E / k T} d E\right],
\end{aligned}
$$

where $r_{M}$ is the classical turning point for $E_{M}$, the value of $E$ at which the integrand on the right hand side reaches a maximum. The integral on the right hand side is the expression (17) used in ${ }^{1} Z_{10}$. In Fig. 14, we plot for the $\mathrm{Cl}_{2}-\mathrm{Cl}_{2}$ system both $\log _{10}\left(Z_{10}{ }^{\mathrm{DWMA}} / Z_{10} \mathrm{EXPA}\right)$ and $\log _{10}\left({ }^{1} Z_{10}{ }^{\mathrm{DWMA}} /{ }^{1} Z_{10}{ }^{\mathrm{EXPA}}\right)$ as a function of $k T / \hbar \omega$. If the fourth approximation is exact, the two curves should be identical. They are different with the greatest disagreement at low temperatures. In Fig. 15 we plot for the $\mathrm{Cl}_{2}-\mathrm{Cl}_{2}$ system $\log _{10}\left(Z_{10}{ }^{\mathrm{EXPA}} / Z_{10}{ }^{\mathrm{LJ}}\right)$ and $\log _{10}\left({ }^{1} Z_{10} \mathrm{EXPA} /\right.$ $\left.{ }^{1} Z_{10} \mathbf{L J}\right)$ as a function of $k T / \hbar \omega$. Because the well affects cross sections less than probabilities, the first curve departs from zero less than the second. In Fig. 16 we plot for the $\mathrm{Cl}_{2}-\mathrm{Cl}_{2}$ system $\log _{10}\left(Z_{10}{ }^{\mathrm{DWMA}} / Z_{10}{ }^{\mathrm{LJ}}\right)$ and $\log _{10}\left({ }^{1} Z_{10}{ }^{\mathrm{DWMA}} /{ }^{1} Z_{10}{ }^{\mathrm{LJ}}\right)$ as a function of $k T / \hbar \omega$. The two curves are very similar, with the greatest departure from zero occurring at low temperatures. For the threedimensional model, Figs. 14 and 15 indicate that the disagreement of $Z_{10}$ DW MA with $Z_{10}{ }^{\mathrm{LJ}}$ at low temperatures is due equally to the inability of SSH theory to correctly include the effects of the well and to accurately infer cross sections from probabilities. We have already shown that ${ }^{1} Z_{10}{ }^{\mathrm{DW} M \mathrm{~A}}$ disagrees with ${ }^{1} Z_{10}{ }^{\mathrm{LJ}}$ at low temperatures due mainly to the effects of the well. If the results shown in Fig. 16 are typical for many collision systems, then our conclusions about the accuracy of ${ }^{1} Z_{10}$ DWMA for the $\mathrm{O}_{2}-\mathrm{O}_{2}$ and the $\mathrm{Br}_{2}-\mathrm{Br}_{2}$ systems could equally well apply to $Z_{10}{ }^{\mathrm{DWMA}}$ for these two systems. To the degree that the modeling described in Sec. II is correct, we can conclude from these calculations that SSH theory accurately predicts collision numbers from known potential parameters except at low temperatures, where the theory incorrectly estimates well effects and cross sections.

SSH theory can also be used to analyze experimental collision numbers for unknown potential parameters.

\footnotetext{
*Present address: Chemistry Division, Argonne National Laboratory, 9700 South Cass Avenue, Argonne, Ill. 60439.

'Alfred P. Sloan Foundation Fellow.

'Contribution No. 4406.

${ }^{1}$ K. F. Herzfeld and T. A. Litovitz, Absorption and Dispersion of Ultrasonic Waves (Academic, New York, 1959), Chap. 7, p. 260.

${ }^{2}$ B. Hartmann and Z. I. Slawsky, J. Chem. Phys. 47, 2491 (1966).

${ }^{3}$ D. J. Wilson, J. Chem. Phys. 54, 540 (1971).

${ }^{4}$ A. F. Wagner and V. McKoy, J. Chem. Phys. 58, 2604 (1972).
}

If we consider ${ }^{1} Z_{10}{ }^{\mathrm{LJ}}$ to be the experimental result, then we can adjust the potential parameters in the SSH theory so that ${ }^{1} Z_{10}$ DWMA becomes equal to ${ }^{1} Z_{10}{ }^{\mathrm{LJ}}$. The resulting potential parameters can then be compared to the $\sigma$ and $\epsilon$ which actually produced ${ }^{1} Z_{10}{ }^{\mathrm{LJ}}$. Let us call $\sigma_{A}$ the potential parameter in conjunction with $\epsilon$ that makes ${ }^{1} Z_{10}{ }^{\mathrm{DWMA}}$ equal to ${ }^{1} Z_{10}{ }^{\mathrm{LJ}}$. Let us call $\epsilon_{A}$ the potential parameter in conjunction with $\sigma$ that makes ${ }^{1} Z_{10}{ }^{\mathrm{DWMA}}$ equal to ${ }^{1} Z_{10}{ }^{\mathrm{LJ}}$. In Fig. 17 , we plot $\left(\sigma_{A} / \sigma\right) \times 100$ and $\left(\epsilon_{A} / \epsilon\right) \times 100$ as a function of $k T / \hbar \omega$ for all three systems. Over this temperature range, $\sigma_{A}$ is within $10 \%-15 \%$ of $\sigma$. However, $\epsilon_{A}$ is never more than $70 \%$ of $\epsilon$, even when $\epsilon$ is very large. For the $\mathrm{O}_{2}-\mathrm{O}_{2}$ system, no $\epsilon_{A}$ can be found which in conjunction with $\sigma$ will make ${ }^{1} Z_{10}$ DW MA equal to ${ }^{1} Z_{10}{ }^{\mathrm{L} J}$. This is so because SSH theory does not properly estimate well effects. Even though $\mathrm{SSH}$ theory is more accurate at high temperatures, it is not because it more accurately predicts well effects, but because well effects are less important at high temperatures. There is no temperature range over which SSH theory will accurately predict the depth of the potential well for our three systems.

\section{CONCLUSIONS}

Three conclusions can be drawn from this work. First, calculations of quantum mechanical cross sections for vibrational scattering off of spherically symmetric potentials are currently practical. Efficient computational methods, suitably restrictive channel sets, and interpolation procedures help make the calculations feasible. Second, the comparison of probabilities and cross sections between the Lennard-Jones potential and several "well-less" potentials emphasizes the adiabatic long-range nature of potentials with wells. The presence of the well washes out structure in the probabilities as a function of energy and produces probabilities and cross sections less than that estimated by the acceleration approximation. Third, SSH theory reasonably accurately predicts collision numbers except at low temperatures where it incorrectly estimates well effects and cross sections. SSH theory can not be used to accurately determine the depth of the well.

${ }^{5}$ V. P. Gutschick and V. McKoy, J. Chem. Phys. 54, 4807 (1970).

${ }^{6}$ D. Secrest and B. R. Johnson, J. Chem. Phys. 45, 4556 (1966).

${ }^{7}$ R. G. Gordon, J. Chem. Phys. 51, 14 (1969).

${ }^{8}$ A. S. Cheung and D. J. Wilson, J. Chem. Phys. 51, 4733 (1969).

${ }^{9}$ J. M. Jackson and N. F. Mott, Proc. R. Soc. A 137, 703 (1932).

${ }^{10}$ F. H. Mies, J. Chem. Phys. 40, 523 (1964).

${ }^{1}$ D. Secrest, J. Chem. Phys. 49, 2880 (1968). 